the vicinity of the epileptic focus. Conversely, DC went on to positive at an area distant from the focus produced. By observing a direction of DC, we could assertain an active area to which surgical manipuiations (cortical excision etc.) should be undertaken.

3) DC changes during a course of asphyxiation produced by cramping the trachea was also observed in immobilized cats. The asphyxial potential named by Leão followed again by a pronounced slowly on-going negative DC shift which amounted more than $10 \mathrm{mV}$. In corresponding roughly with the stage of negativegoing DC shift, evoked visual cortical response elicited by stimulating the optic tract went out completely and no cases showed a reappearance of ECoG by releasing obstruction of the trachea.

It was concluded that pronounced slowly-going DC shift reflected a critical stage regarding the irreversible loss of a function of the brain.

\title{
F-7. Somatosensory Evoked Responses in Patients with Lesion of Nervous System
}

\author{
Yutaka Hori, Shozaburo Utsumi, Chikayoshi Terada, \\ Atsuo Okazaki, Masatsugu Kobatake and Ryoichi Odani \\ The Second Department of Surgery, Nara Medical Uniuersity
}

There have not been many studies concerning somatosensory evoked responses (SERs) in patients, and the significance of the each component of the SERs has not been satisfactorily clarified on neurophysiological basis. In order to evaluate the abnormal SERs, studies have been made on 182 neurosurgical cases and SERs have been reviewed from the points of clinical application.

SERs were recorded at a point of $2.5 \mathrm{~cm}$ posterior and $7 \mathrm{~cm}$ lateral of the vertex, following the cathodal electrical stimulation, $0.2 \mathrm{msec}$. pulse duration, at a rate of 1 per second, on contralateral median nerve at wrist. A total of 100 or 200 responses were summated. Usually responses were tested at the intensity of motor threshold.

The components of SERs consisted of about seven waves within the first 250 msec. following application of the stimuli.

1) In peripheral nerve lesions, SERs were diminished with impaired proprioceptive sense but not affected with impaired exteroceptive sense as well as motor weakness.

2) SERs were absent or remarkably affected in posterior cervical lesions and less affected in anterior cervical lesions.

3) In cases of diffuse brain stem injuries, the late components of SERs (wave $5,6,7)$ were affected. In the mild cases they were inhibited and showed prolongation of latencies. In moderate disturbances they showed big synchronized waves, and disappeared in advanced disturbances. 
4) In cortical sensory and motor lesions, the early components of SERs (wave $1,2,3)$ wer diminished in amplitude, showing their prolongation in latencies.

5) SERs would completely disappear in an extensive and advanced injuries of the cortex as well as in brain stem lesion with coma.

6) In the all cases of centrencephalic epilepsy, SERs showed conspicuously large negative wave with the peak around 170-200 msec. in latency, which were the characteristic late components bilaterally.

7) Wave 4 was inhibited in disturbances of motor area and would be well correlated with potentials of somatomotor cortex.

Clinical application of SERs would be great use combining with neurological and electroencephalographical findings.

\title{
F-8. Clinical Anplication of Somatosensory Evoked Potential Method in Neurosurgical Cases
}

\author{
Noboru Sakai, Keiichi Misawa and Kazuki Sakata \\ Second Department of Surgery, Gifu University School of Medicine
}

Clinical application of somatosensory evoked potnetial (SEP) examination in neurosurgical cases was studied. Bilateral ulnar nerves were simultaneously stimulated and bilateral SEPs were recorded bipolarly from the scalp surfaces correlsponding to somatosensory areas. Peak latency and ratio of amplitude between both sides were measured on various components of SEP in 33 normal subjects and in 45 cases of various organic brain diseases. Results obtained were as follows.

1) Forty-five cases of various orgaic brain diseases were classified into tumor $(\mathrm{T})$, hematoma $(\mathrm{H})$ and cerebrovascular lesion (CVL) groups. Peak latency tended to be prolonged in all 3 groups, as compared with normal control. This tendency was marked on the pathological side and, in T-group, it was especially marked in late components. Ratio of amplitude on the lesion side to that on the healthy side showed the following tendency. It was often larger than 1.0 in $\mathrm{N}_{1}$ and smaller than 1.0 in $\mathrm{N}_{2}$ in $\mathrm{T}$-group. In the other 2 groups, it was smaller than 1.0 in both $\mathrm{N}_{1}$ and $\mathrm{N}_{2}$, except that in some cases of chronic subdural hematoma the ratio of amplitude in $\mathrm{N}_{2}$ was almost equal to or larger than 1.0 .

2) T-group was divided into malignant and benign subgroups on the histological ground. Tendency of peak latency delay and tendencyof higher amplitude of $\mathrm{N}_{1}$ on the lesion side were more marked in the malignant subgroup.

3) From the point of view of tumor localization, SEP changes were most marked in the cases of parieto-temporal or thalamic tumors and no SEP change was observed in the cases of tumors in the chiasmal region.

4) In the cases of unilateral sensory disturbance of central origin, peak latencies of $N_{1}$ and $N_{2}$ tended to be prolonged and reduction in amplitudes of $N_{1}$ and $N_{2}$ (especially $\mathrm{N}_{1}$ ) on the lesion side was characteristic, except that in a few tumor cases 